\title{
Localized Memories in Idiotypic Networks
}

\author{
Gérard Weisbuch $\dagger$, Rob J. De Boer $\ddagger$ and Alan S. Perelson $\ddagger \S$
}

† Laboratoire de Physique Statistique de l'Ecole Normale Supérieure, 24 rue Lhomond, F75231 Paris Cedex 5, France, $\ddagger$ Theoretical Division, Los Alamos National Laboratory, Los Alamos, NM 87545, U.S.A. and § The Santa Fe Institute, 1120 Canyon Road, Santa Fe, NM 97501, U.S.A.

\section{(Received on 11 October 1989, Accepted in revised form on 6 April 1990)}

The present paper investigates conditions under which immunological memory can be maintained by stimulatory idiotypic network interactions. The paper was motivated by the work of (De Boer \& Hogeweg, 1989b, Bull. math. Biol. 51, 381-408.) which claimed that idiotypic memory is not possible because of percolation within the network.

Here we reinvestigate the issue of percolation using both the previous model and a simpler one (Weisbuch, 1990, J. theor. Biol. 143, 507-522.) that allows analytic analysis. We focus on network topologies in which each Ab1 is connected to several $\mathrm{Ab} 2 \mathrm{~s}$, which in turn are connected to several Ab3s. It is demonstrated that, for a considerable range of parameters, both models account for the existence of localized memory-states in which only the $\mathrm{Ab} 1$ and the $\mathrm{Ab} 2$ clones are activated and the clones of the Ab3 level remain virgin.

The existence of localized memory-states seems to contradict the previous percolation result. This discrepancy will be shown to depend on the system dynamics. By simulation we explore the parameter regimes for which one finds percolation and those for which localized memory-states exists. We show that the conditions required for attaining the localized memory-state are considerably more stringent than those required for its existence and local stability. We conclude that both localized memory and percolation are possible in stimulatory idiotypic networks.

\section{Introduction}

One of the most striking features of the immune system is its ability to respond in an enhanced manner to antigens which it has previously seen. This phenomenon is called immune memory (or simply immunity) and is the rationale behind vaccination. Despite the fact that vaccination has been used for 200 years, the cellular or molecular basis of memory remains an open question. The hypothesis that we focus on here is that immunological memory is a dynamical consequence of network interactions. The opposing view is that memory is due to the persistence of long-lived memory cells, antigen, and/or circulating antibodies.

Jerne (1974) postulated that the immune system functions as a network with a complexity comparable to that of the neural network. The idea of an immune network is based upon "idiotypic" interactions between lymphocytes expressing receptors of complementary shapes. In this view, antigen is recognized by clones called $\mathrm{Ab1}$, which in turn interact with clones called $\mathrm{Ab} 2$, which in turn interact with other clones denoted $\mathrm{Ab3}$, and so on. Jerne postulated that the network is 
maintained in a steady-state due to a combination of stimulatory and suppressive interactions between clones. To ensure homeostasis suppression was proposed to be the dominating influence. In this view, perturbation of the network with antigen would release clones from network suppression. If after the response, the network settled down to a new steady-state in which these clones were more responsive to antigen, immunological memory would result. Mathematical studies of Jerne's ideas followed rapidly (Hoffmann, 1975; Richter, 1975). Both adopted the "release of suppression" idea, and both demonstrated the existence of several attractors corresponding to states of immunity or suppression.

Richter $(1975,1978)$ suggested the idea of a chain reaction. If an antigen stimulates clones $\mathrm{Ab} 1$, which in turn stimulate clones $\mathrm{Ab} 2$, and so on, a chain reaction could occur in which the response to antigen could activate the entire immune system. Having suppression dominate stimulation, as postulated by Jerne, was meant to cure this potential problem. However, if Richter's model is extended with a small source and a decay term, chain reactions can occur despite the dominance of suppression (Hiernaux, 1977; and our unpublished results).

Hoffmann et al. (Hoffmann, 1975, 1979, 1980; Gunther \& Hoffmann, 1982; Hoffmann et al., 1988) also developed "release of suppression" models. The basic idea of these models is to consider only the Ab1 and the Ab2 populations, which were called "plus" and "minus". The model accounts for four attractors in each of which the degree of suppression between the plus and minus clones differs. Because these models only considered the plus and the minus clone, chain reactions were not possible. The extension of these models into networks involving a large number of clones is currently being developed (Hoffmann et al., 1988).

A new class of network models has been developed by De Boer (1988), Perelson (1988, 1989a), Varela et al. (1988), De Boer \& Hogeweg (1989a, b, c), De Boer et al. (1990), and Weisbuch (1990). These models rely only upon stimulatory network interactions; a decrease in the amplitude of stimulation plays the role of suppression. Stimulatory interactions can also account for dynamic memory. Reciprocal stimulation between $\mathrm{Ab} 1$ and $\mathrm{Ab} 2$ clones maintain an elevated level of $\mathrm{Ab} 1$ clones triggered by antigen. This paper will address Richter's potential chain reaction in these models.

One can either use percolation theory (Perelson, 1989a,b) or theory of random graphs (De Boer \& Hogeweg, 1989b) to show that the graph representing the connections in an idiotypic network probably is one large connected component. Hence, if a chain reaction develops it is expected to affect the entire network. Additionally, the prevention of the chain reaction can only come from the dynamical interactions between the clones, and not from the topology.

The problem of immunity and the chain reaction in networks with only stimulatory interactions was analyzed by De Boer $(1988,1989)$ and De Boer \& Hogeweg $(1989 a, b, c)$. These authors first showed that a stimulatory idiotypic interaction between an Ab1 and an Ab2 clone accounts for a stable immune-state in which an elevated Ab2 clone maintains the stimulation of an even larger Ab1 clone (De Boer, 1988; De Boer \& Hogeweg, 1989a). Then they went on to show that if the Ab2 clone is to maintain the proliferation of the Ab1 clones, it is also expected to stimulate clones on the Ab3 level (De Boer, 1989; De Boer \& Hogeweg, 1989b). 
The Ab3 clones in turn activate the Ab4 level, and so on. They identified this as the "percolation problem", and argued that idiotypic memory should not be possible.

This percolation problem was subsequently investigated by Weisbuch $(1989,1990)$. First, he showed that discrete threshold automata, that have simple suppressive and stimulatory idiotypic interactions as inputs, account for organized behavior provided the network is sufficiently dilute (Weisbuch, 1989). These simple networks remain functionally disconnected into quasi-independent subunits. This would solve the percolation problem. Second, he analysed a piece-wise linear form of De Boer \& Hogeweg's model. Analytical results showed that whenever the connection strength between the $\mathrm{Ab} 2$ and $\mathrm{Ab} 3$ clones remained sufficiently weak, percolation did not occur and memory remained localized with only clones at the Ab1 and Ab2 levels activated (Weisbuch, 1990).

The present paper, motivated by a confrontation of the opposite conclusions reached by De Boer \& Hogeweg (percolation) and Weisbuch (localization), resolves the issue. We show that both views are correct: a network can generate localized memory in some parameter regimes, while in other parameter regimes percolation destroys the reasonable dynamic behavior of the network.

We examine the possibility of idiotypic memory in situations where each clone has several idiotypic interactions with other clones. This we believe is indicative of the general biological situation. Following Weisbuch (1990), we introduce a piecewise-linear expression that describes the rate of proliferation of a clone. This function allows us to derive algebraic conditions for the existence of stable localized memory. The existence of localized memory is then verified for the more "biological" model (De Boer, 1988; De Boer \& Hogeweg, 1989a, b) in which a log bell-shaped activation function is used. It will be shown that depending upon parameter settings both localized memory and percolation are possible in this model. We thus conclude that idiotypic network models based upon stimulatory interactions can enclode immunological memory.

\section{Simple Stimulatory Network Models}

We (De Boer, 1988; De Boer \& Hogeweg, 1989a, b; Weisbuch, 1990) have previously proposed two very similar models. The models specify clones of Blymphocytes that (1) are formed in the bone marrow, (2) proliferate due to idiotypic stimulation, and (3) die in the periphery. For each clone " $i$ " we consider the total amount of anti-idiotypic stimulation as a linear combination of all other clones " $j$ ". We call $h_{i}$ the field of clone $x_{i}$ :

$$
h_{i}=\sum_{j} J_{i j} x_{j}
$$

where $J_{i j}$ specifies the affinity between clone $x_{i}$ and $x_{j}$. For reasons of simplicity, $J_{i j}=0$ (no interaction) or 1 (maximum interaction) here. The distribution of zero's and one's in the $J$ matrix defines the topology of the network. Although we envisage the influence of clone $j$ on clone $i$ to be mediated by antibody, we do not explicitly include antibody in this model. 
The clone sizes change in time according to the system of differential equations:

$$
\frac{\mathrm{d} x_{i}}{\mathrm{~d} t}=s+x_{i}\left[f\left(h_{i}\right)-\mu\right],
$$

where $s$ is the source of cells from the bone marrow, the function $f\left(h_{i}\right)$ defines the rate of proliferation, and $\mu$ specifies the rate of cell death.

The function $f\left(h_{i}\right)$ is non-negative. The model thus only incorporates the possibility of cell growth due to idiotypic interactions. Nevertheless, we describe $f\left(h_{i}\right)$ as having both a stimulatory and a suppressive part. Within the stimulatory part increasing the idiotypic field increases proliferation; within the suppressive part increasing the field decreases proliferation. Similar dose response curves are found in receptor crosslinking (Perelson, 1984). We here use a piecewise linear and a log bell-shaped $f\left(h_{i}\right)$ function (see Fig. 1). Both have a threshold for activation (i.e. $\theta_{1}$ ) and one for suppression (i.e. $\theta_{2} / 2$ or $\theta_{2}$ ). The piecewise linear function facilitates mathematical treatment, the log bell-shaped function is more realistic and allows us to test the robustness of the results obtained with the piecewise linear function.

Figure 1(a) displays the piecewise linear function which has four regions

$$
f(h)=\left\{\begin{array}{ll}
0 & \text { if } h \leq \theta_{1} \\
h-\theta_{1} & \text { if } \theta_{1}<h \leq \theta_{2} / 2 \\
\theta_{2}-\theta_{1}-h & \text { if } \theta_{2} / 2<h \leq \theta_{2}-\theta_{1} \\
0 & \text { if } h>\theta_{2}-\theta_{1}
\end{array} .\right.
$$

This function is very similar to the one defined previously by Weisbuch (1990). However, the previous function did not incorporate the fourth, zero region.

Figure 1(b) displays the $\log$ bell-shaped $f(h)$ function, with a maximum rate of proliferation $p$. This function was defined previously by De Boer (1988) and De

$f(h)$

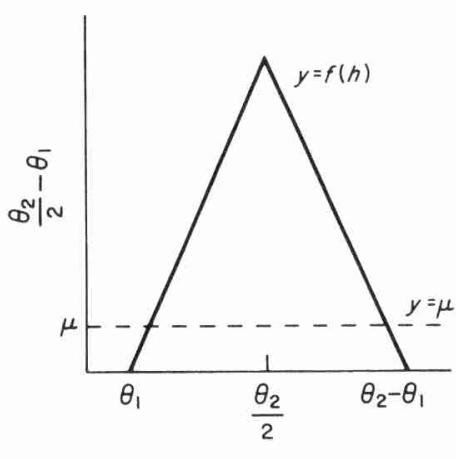

$h$ (a) $\quad f(h)$

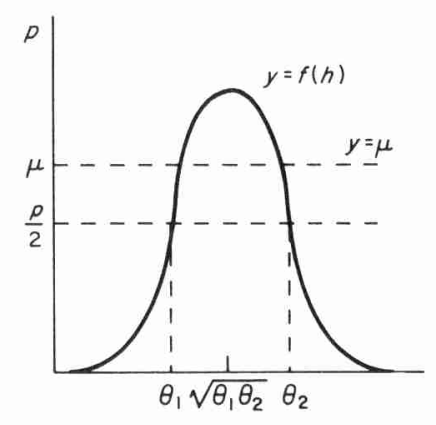

$\log h$

(b)
FIG. 1. Graphs of the piecewise linear (a) and the log bell-shaped (b) proliferation function $f(h)$ vs. $h$ [eqns (3) and (4)]. $h$ is the field acting upon the clone due to idiotypic stimulation. $f(h)$ is the rate of proliferation per cell per day. Equilibria involving proliferation are close to the intersections of $y=f(h)$ and $y=\mu$ (see the text). 
Boer \& Hogeweg (1989a). It is based upon the product of two functions, one increasing from zero to one, and one decreasing from one to zero, with half saturation constants $\theta_{1}$ and $\theta_{2}$, respectively. For $\theta_{2} \gg \theta_{1}$, this $f(h)$ function can be approximated by its ascending or stimulating part for $h \ll \theta_{2}$, and by its descending part for $h \gg \theta_{1}$. When $f(h)$ is plotted vs. $\log h$ it is bell-shaped and symmetric around its maximum at $h=\sqrt{\theta_{1} \theta_{2}}$. The log bell-shaped function is:

$$
f(h)=p \frac{h}{\theta_{1}+h} \frac{\theta_{2}}{\theta_{2}+h} .
$$

The piecewise linear and the log bell-shaped functions are not meant to be direct analogs of one another, they have different heights and characteristic widths. But as we show in the next section, they generate the same types of steady-state behavior.

\section{Two Clone Results}

We here summarize previous results (De Boer, 1988; De Boer \& Hogeweg, 1989a; Weisbuch, 1990). In the absence of idiotypic interactions (i.e. $h_{i}=0$ ) the differential eqn (2) has one stable equilibrium. This is the virgin-state located at

$$
x_{i}=\frac{s}{\mu} .
$$

If two virgin clones connected by an idiotypic interaction are to remain virgin the following condition must hold:

$$
\frac{s}{\mu}<\theta_{1}
$$

When clones are proliferating, i.e. $x_{i} \gg s / \mu$, we can easily predict their equilibrium value by neglecting, in a first approximation, the source term. This makes sense biologically because for each clone the number of cells supplied by the bone marrow should be small compared to the number generated by the proliferation of a stimulated clone. With $s \approx 0$, proliferating clones can be in equilibrium only if proliferation and decay balance, i.e. if

$$
f(h) \approx \mu .
$$

Examining the curves $y=f(h)$ and $y=\mu$ in Fig. 1, one observes that these curves intersect twice, i.e. once in the stimulatory and once in the suppressive region of $f(h)$. This corresponds to two possible equilibrium-states for each clone. We call the equilibrium in the stimulatory region the immune-state; in this state further activation increases proliferation. We call the equilibrium in the suppressive region the suppressed-state; here the clone is partially suppressed, i.e. further activation decreases proliferation. Thus, depending on the field value $\left(h_{i}\right)$, there are three different equilibria for each clone: virgin, immune and suppressed. 
The location of these equilibria in the plane of the two populations $\left(x_{1}\right.$ and $\left.x_{2}\right)$ is shown in Fig. 2. Only three equilibrium configurations among the five intersections are stable:

-one attractor in which both clones are in the virgin-state [eqn (5)];

-two attractors, one with clone $x_{1}$ in the immune-state and $x_{2}$ in the suppressedstate and the other with $x_{2}$ in the immune-state and $x_{1}$ in the suppressed-state [both satisfy eqn (7)].

States with both clones immune or both suppressed are unstable. Figure 2 shows that both models [i.e. eqn (3) and eqn (4)] have these same types of equilibriumstates.
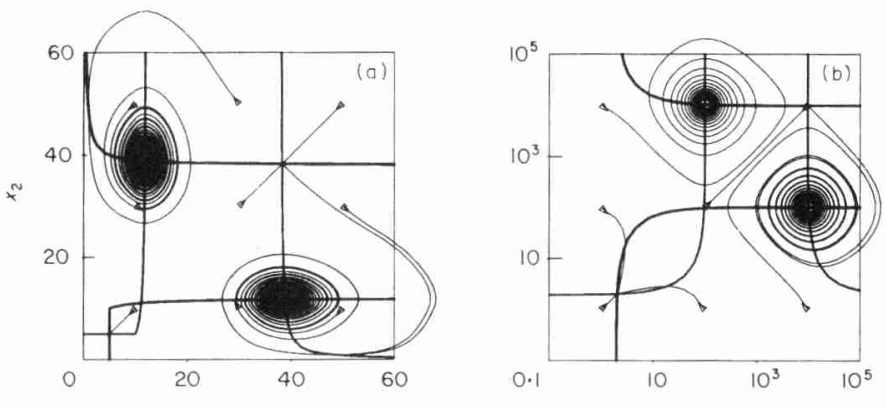

FIG. 2. Trajectories (light lines starting with an arrowhead) and nullclines (heavy lines) of the idiotypic interaction between two clones, $x_{1}$ and $x_{2}$, for the picewise linear (a) and the log bell-shaped (b) $f(h)$ function respectively. The trajectories settle into the virgin, the immune, and the suppressed attractors for $x_{1}$. Two trajectories starting at the diagonal line $x_{1}=x_{2}$ seem to settle into one of the two unstable intersects of the null clines. However, due to the instability they both escape, and settle into the immune-state of $x_{1}$. Parameter setting in (a): $s=10, \theta_{1}=10, \theta_{2}=50, \mu=2$; in (b): $s=1, \theta_{1}=10^{2}, \theta_{2}=10^{4}$, $p=1, \mu=0 \cdot 5$. Isoclines and numerical integration was performed by means of GRIND (De Boer, 1983).

\section{Topology of Interactions in Large Networks}

Let us now consider the dynamics of a network composed of a large number of interacting clones which obey eqn (2).

Consider a random network with an average of $m$ connections per clone. Such a network can be approximated by a lattice in which each clone has exactly $m$ connections. Such lattices are called Bethe lattices, and $m$ is called the co-ordination number. In this paper we consider for simplicity only the situation where introduction of an antigen elicits a response from only a single clone. The introduction of such an antigen selects this clone as a root from where stimulation will spread over the lattice. This root is called the Ab1 clone. The presence of a root converts the lattice into a tree like structure called a Cayley tree with each node having degree $\mathrm{m}$. We plot a number of different Cayley trees in Fig. 3(b).

The tree topology does not take into account the possible existence of loops in the net. However, this is of no importance when the sub-net of excited clones does not contain loops. If short loops exist, the mathematical treatment given here has 
(a)

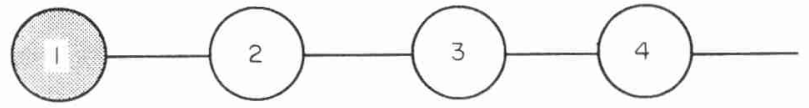

(b)
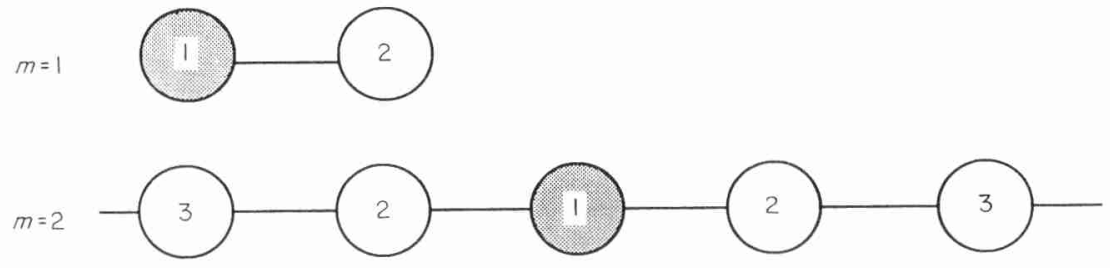

$m=3$

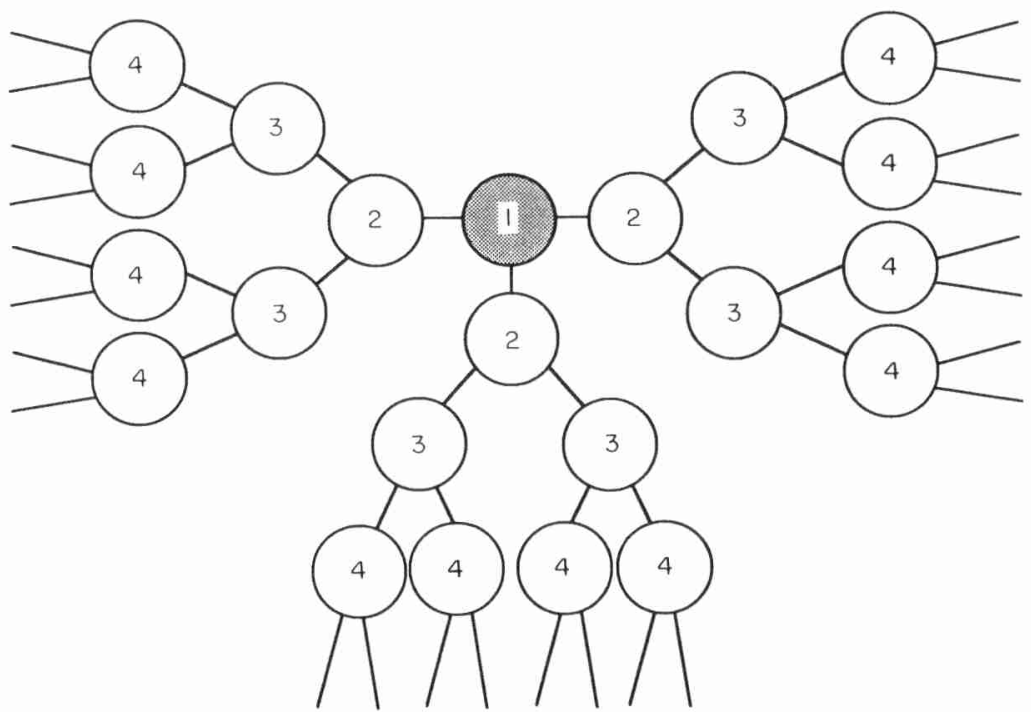

FIG. 3. Network topologies. (a) The symmetric linear Ab1, ., Abn chain. (b) Cayley tree topologies with respectively 1,2 , and 3 symmetric connections per clone. The darkened circle, Ab1, is the root of the chain in (a) and each tree in (b).

to be slightly modified (see section 7), but the conclusion concerning the possible existence of localized memory in some range of parameters remains the same.

Consider a Cayley tree in which each clone has $m$ neighbors. The fields of clones $\mathrm{Ab} 1, \mathrm{Ab} 2, \mathrm{Ab} 3$ are

$$
\begin{aligned}
& h_{1}=m x_{2}, \\
& h_{2}=x_{1}+(m-1) x_{3}, \\
& h_{3}=x_{2}+(m-1) x_{4} .
\end{aligned}
$$


In general

$$
h_{i}=x_{i-1}+(m-1) x_{i+1}, \quad i>1 .
$$

Anticipating our later results, we point out the importance of having a branching tree rather than the commonly used sequence $\mathrm{Ab} 1 \leftrightarrow \mathrm{Ab} 2 \leftrightarrow \mathrm{Ab} 3 \leftrightarrow \mathrm{Ab} 4 \ldots$ displayed in Fig. 3(a). To obtain immunity, we require that clone Ab2 strongly stimulates Ab1 so as to excite it into the immune region. Concomitantly, to avoid percolation, we require the stimulation of $\mathrm{Ab} 3$ by $\mathrm{Ab} 2$ to be sufficiently weak that $\mathrm{Ab} 3$ is not excited. For this to occur, $h_{3}$ must be smaller than $h_{1}$, which, according to eqns (8a) and (8c), is only possible if $m$ is strictly larger than one (if $m=1, h_{3}=h_{1}$ ). For the commonly used sequence displayed in Fig. 3(a), $h_{1}=x_{2}, h_{2}=x_{2}+x_{3}, h_{3}=x_{2}+x_{4}$. The field $h_{1}$ cannot be larger than $h_{3}$; thus localized memory is not possible in this case.

Note that although all idiotypic interactions are symmetric (i.e. all $J_{i j}=J_{j i}$ ), antigen generates an asymmetry between the $\mathrm{Ab} 1$ and $\mathrm{Ab} 2$ level (i.e. one $\mathrm{Ab} 1$ interacts with $m$ Ab2s).

\section{A Simple Piecewise Linear Three Species System}

Let us search for solutions to eqn (2) which display localized memory around Ab1. In order for the memory to be localized, we look for solutions in which all populations above the Ab2 level are in the virgin-state (i.e. $x_{i}=s / \mu$, for $i=3$, $4, \ldots, n)$. The model then reduces to

$$
\begin{aligned}
& \frac{\mathrm{d} x_{1}}{\mathrm{~d} t}=s+x_{1}\left[f\left(m x_{2}\right)-\mu\right], \\
& \frac{\mathrm{d} x_{2}}{\mathrm{~d} t}=s+x_{2}\left[f\left(x_{1}+\frac{(m-1) s}{\mu}\right)-\mu\right] .
\end{aligned}
$$

If solutions to eqns (10) and (11) can be found with $x_{2}$ sufficiently small to maintain $x_{3}$ in the virgin-state, then we have found a localized memory-state. According to the sizes of the fields acting on $x_{1}$ and $x_{2}$, relative to the two thresholds $\theta_{1}$ and $\theta_{2} / 2$, the $x_{1}-x_{2}$ plane can be divided into 16 regions in each of which $f$ is a linear function of $x_{1}$ and $x_{2}$. In the solution that we are looking for, Ab1 should suppress the proliferation of the Ab2s, whereas the Ab2s should sustain the proliferation of $\mathrm{Ab} 1$. In these regions the $f$ function of the $\mathrm{Ab} 1$ is $h-\theta_{1}$, and that of the Ab2 is $\theta_{2}-\theta_{1}-h[$ eqn (3)]. Equations (10) and (11) then simplify into

$$
\begin{aligned}
& \frac{\mathrm{d} x_{1}}{\mathrm{~d} t}=s+x_{1}\left(m x_{2}-\theta^{\prime}\right), \\
& \frac{\mathrm{d} x_{2}}{\mathrm{~d} t}=s+x_{2}\left(\theta^{\prime \prime}-x_{1}\right),
\end{aligned}
$$

where

$$
\theta^{\prime}=\mu+\theta_{1} \quad \text { and } \quad \theta^{\prime \prime}=\theta_{2}-\theta_{1}-\frac{(m-1) s}{\mu}-\mu .
$$


Linear analysis in the vicinity of the equilibrium point $\dot{x}_{1}=0$ and $\dot{x}_{2}=0$ only involves solving quadratic equations. The stationary point co-ordinates $\left(X_{1}, X_{2}\right)$ are

$$
\begin{aligned}
& X_{1}=\frac{s(1+m)+\theta^{\prime} \theta^{\prime \prime}+\sqrt{\left[s(1+m)-\theta^{\prime} \theta^{\prime \prime}\right]^{2}+4 s m \theta^{\prime} \theta^{\prime \prime}}}{2 \theta^{\prime}}, \\
& X_{2}=\frac{-s(1+m)+\theta^{\prime} \theta^{\prime \prime}+\sqrt{\left[s(1+m)-\theta^{\prime} \theta^{\prime \prime}\right]^{2}+4 s m \theta^{\prime} \theta^{\prime \prime}}}{2 m \theta^{\prime \prime}} .
\end{aligned}
$$

Consider the case of a small source (i.e. $m s \ll \theta^{\prime} \theta^{\prime \prime}$ ), which we consider the most biologically meaningful. In this case, eqn (15) can be approximated by the following series:

$$
\begin{aligned}
& X_{1} \approx \theta^{\prime \prime}+\frac{m s}{\theta^{\prime}}+\frac{m s^{2}}{\theta^{\prime 2} \theta^{\prime \prime}}+\ldots, \\
& X_{2} \approx \frac{\theta^{\prime}}{m}-\frac{s}{m \theta^{\prime \prime}}+\frac{s^{2}}{\theta^{\prime} \theta^{\prime \prime 2}}+\ldots .
\end{aligned}
$$

Above we argued that with a small source equilibrium was obtained when cell proliferation and death balanced, i.e. $f(h) \approx \mu$ [see eqn (7)]. This can now easily be verified. For Ab1, $h_{1}=m X_{2}$. Thus ignoring terms of $\mathrm{O}(s), f\left(h_{1}\right)=f\left(\theta^{\prime}\right)=$ $f\left(\theta_{1}+\mu\right)$, which equals $\mu$ by eqn (3). Similarly for $\mathrm{Ab}_{2}, h_{2}=X_{1}+(m-1) s / \mu \approx$ $\theta_{2}-\theta_{1}-\mu$. Again, by eqn (3), $f\left(h_{2}\right)=\mu$, assuming $\theta_{2}-\theta_{1}-\mu>\theta_{2} / 2$.

Local stability analysis determines whetier this $X_{1}-X_{2}$ equilibrium is stable. The partial derivatives of eqns (12) and (13) give the Jacobian matrix

$$
\left[\begin{array}{cc}
m X_{2}-\theta^{\prime} & m X_{1} \\
-X_{2} & \theta^{\prime \prime}-X_{1}
\end{array}\right]
$$

The eigenvalues $\lambda$ obey the characteristic equation

$$
\lambda^{2}+\lambda\left(\theta^{\prime}-m X_{2}+X_{1}-\theta^{\prime \prime}\right)+X_{1} \theta^{\prime}+m X_{2} \theta^{\prime \prime}-\theta^{\prime} \theta^{\prime \prime}=0
$$

with solutions to order $s$

$$
\lambda_{ \pm} \approx \frac{-s\left(m \theta^{\prime \prime}+\theta^{\prime}\right)}{2 \theta^{\prime} \theta^{\prime \prime}} \pm i \sqrt{\theta^{\prime} \theta^{\prime \prime}}\left[1+\frac{(m-1) s}{2 \theta^{\prime} \theta^{\prime \prime}}\right] .
$$

The eigenvalues have a small negative real part. The imaginary part is large. We conclude that the stationary point [eqns (14) and (15)] is an attractor, and that the slow convergence towards the attractor is oscillatory. Trajectories are very similar to those in Fig. 2(a) (in which $m=1$ ).

To conclude that we have found a localized memory-state we need to verify that the attractor is indeed in the correct region of the $x_{1}-x_{2}$ plane and that $x_{3}$ remains virgin. Since $\mathrm{Ab} 2$ should be suppressed we require $\frac{1}{2} \theta_{2}<h_{2}<\theta_{2}-\theta_{1}$, or

$$
\theta_{2}>2\left(\theta_{1}+\mu\right) \text {. }
$$

Since Ab3 should remain virgin, we require $h_{3}<\theta_{1}$, or

$$
X_{2}+\frac{(m-1) s}{\mu}<\theta_{1} \text {. }
$$


Using eqn (17) this can be approximated by

$$
\frac{\mu}{m-1}+\frac{m s}{\mu}<\theta_{1} .
$$

This condition implies that $m s / \mu<\theta_{1}$, i.e. a virgin clone surrounded by $m$ virgin neighbors should not be exposed to a field larger than $\theta_{1}$. The other inequalities that ensure that $\mathrm{Ab} 1$ should remain immune, i.e. $\theta_{1}<h_{1}<\theta_{2} / 2$, are less restrictive and are therefore omitted. In conclusion, we end up with two parameter conditions [eqns (21) and (23)] for the existence of a stable equilibrium in which Ab1 is kept immune by $\mathrm{Ab} 2 \mathrm{~s}$ under conditions where Ab3 remains virgin.

Immunologically, both conditions seem likely to be fulfilled since the threshold for suppression should be much larger than that for stimulation [i.e. $\theta_{2} \gg \theta_{1}$, condition (21)] and since the source of cells from the bone marrow [ $s$, condition (23)] is small for each clone. The model thus accounts for a localized memory-state in a wide range of parameter settings.

The equilibrium eqns (16) and (17) were verified numerically by starting close to the predicted equilibrium. Parameter values that do not obey the inequalities (21) and (23) indeed fail to give rise to localized memory.

\section{The Log Bell-shaped Activation Function}

The piecewise linear function [eqn (3)] is only a useful mathematical approximation. Two of its unrealistic features require verification, which can be done by using the log bell-shaped function [eqn (4)]. The fact that the equilibrium derived above [i.e. eqns (14) and (15)] is stable might depend on the assumption that below $\theta_{1}$ proliferation is exactly zero (Fig. 1). A smooth transition from zero to slow proliferation might destablize the virgin-state of an Ab3. Secondly, an artefact of the piecewise linear function is that cells may proliferate very fast, since the maximum of the function is determined by $\theta_{1}$ and $\theta_{2}$.

For the bell-shaped function it is relatively easy to find realistic parameter values. B cells have a life-time of $1-2$ days which corresponds to $0 \cdot 5 \leq \mu \leq 1 \cdot 0$ day $^{-1}$. Cell division takes about $16 \mathrm{hr}$. Using the bell-shaped function the maximum net rate of proliferation is $p-\mu$ [see eqns (2) and (4)]. We therefore take $0 \cdot 5 \mathrm{day}^{-1}$ as a typical value of $p-\mu$. The production of naive B cells in the bone marrow is $10^{6}-10^{7}$ cells day $^{-1}$. Since this covers more than $10^{6}$ different specificities, the source for each clone, $s$, should be around one cell day ${ }^{-1}$. If $0.5 \leq \mu \leq 1.0$ day $^{-1}$ the average virgin clone size, $s / \mu$, is about two cells, which is reasonable (Jerne, 1984). The two thresholds $\left(\theta_{1}\right.$ and $\left.\theta_{2}\right)$ should be several orders of magnitude apart to allow for considerable clonal expansion [we generally choose for $\theta_{1}=10^{2}$ and $\theta_{2}=10^{4}$, see e.g. Fig. 2(b)].

In analogy with the derivation of the algebraic conditions for the existence of localized memory in the piecewise linear model, we derive conditions for localized memory in the log-bell-shaped model. For small $s$, approximate steady-state values for $X_{1}$ and $X_{2}$ are obtained at the intersections of the $f(h)$ curves with $\mu$. Since $\mathrm{Ab} 2$ is suppressed in the localized memory-state, $h_{2} \gg \theta_{1}$, and we approximate $f\left(h_{2}\right)$ 
by its descending part, i.e.

$$
\mu=f\left(h_{2}\right)=p \frac{h_{2}}{\theta_{1}+h_{2}} \frac{\theta_{2}}{\theta_{2}+h_{2}} \approx p \frac{\theta_{2}}{\theta_{2}+h_{2}},
$$

where

$$
h_{2}=X_{1}+\frac{(m-1) s}{\mu} .
$$

Solving for $X_{1}$ gives

$$
X_{1} \approx \frac{(p-\mu) \theta_{2}}{\mu}-\frac{(m-1) s}{\mu} .
$$

Similarly, since Ab1 is activated in the localized memory-state, $h_{1} \ll \theta_{2}$, and we approximate $f\left(h_{1}\right)$ by its ascending part. Thus, at steady-state

$$
\mu=f\left(h_{1}\right) \approx p \frac{h_{1}}{h_{1}+\theta_{1}} \quad \text { where } h_{1}=m X_{2} .
$$

Solving for $X_{2}$ we find that

$$
X_{2} \approx \frac{\mu \theta_{1}}{m(p-\mu)} .
$$

Again the conditions for the existence of localized memory imply that Ab3 clones, even when stimulated, fail to proliferate. Thus, $h_{3}<\theta_{1}$ and we approximate $f\left(h_{3}\right)$ by its ascending part:

$$
f\left(h_{3}\right) \approx p \frac{h_{3}}{h_{3}+\theta_{1}}<\mu, \text { where } h_{3}=X_{2}+\frac{(m-1) s}{\mu} .
$$

When eqn (25b) is substituted for $X_{2}$ we find,

$$
\frac{m s}{\mu}<\theta_{1} \frac{\mu}{(p-\mu)} .
$$

Since $\mu /(p-\mu)$ is of order one, this is comparable to eqn (23), i.e. it only requires the existence of the virgin-state. The main result of the piecewise linear model, i.e. the existence of the localized memory-state, can thus be generalized to the bellshaped model.

\section{Networks with Short Loops}

The above analysis should also apply to networks with short loops. To understand this, consider the case of a periodic lattice where each node has a co-ordination number $m$. The fields on $\mathrm{Ab} 1$ and $\mathrm{Ab} 3$ are:

$$
\begin{aligned}
& h_{1}=m x_{2}, \\
& h_{3}=\alpha m x_{2}+(1-\alpha) m x_{4},
\end{aligned}
$$


where $\alpha<1$. On a square lattice, e.g. an Ab3 has either one or two Ab2 neighbors; therefore $\alpha=0.5$ or 0.25 . Since $\alpha m x_{2}$ [eqn (28b)] is smaller than $m x_{2}$ [eqn (28a)], it is always possible to find a range of parameters such that $h_{1}$ is above $\theta_{1}$ (and $x_{1}$ is excited), while $h_{3}$ is below $\theta_{1}$ (and $x_{3}$ remains virgin).

\section{Dynamic Analysis}

The present results illustrating localized memory seem to contradict the previous analysis in which the percolation problem was identified (De Boer \& Hogeweg, $1989 \mathrm{~b}$ ). This is surprising since the previous analysis also included idiotypic stimulation of one Ab1 by several Ab2s, hence, similar results would have been expected. This apparent discrepancy turns out to be due to the system dynamics.

The mere existence of certain stable attractors (here localized memory-states) does not imply that they are actually attained from other attractors following certain perturbations. Thus the following question arises. What are the conditions that assure that the localized memory-state will be attained following a perturbation of the virgin-state? The previous claim (De Boer \& Hogeweg, 1989b) was that one either observes percolation or a return to the virgin-state following such a perturbation.

To answer this question for the present model, we incorporate the dynamics of the immune response by extending the bell-shaped model with a simple equation describing antigen removal:

$$
\frac{\mathrm{d} a}{\mathrm{~d} t}=-\operatorname{kax}_{1} .
$$

The field of the Ab1 [eqn (8)] now becomes:

$$
h_{1}=m x_{2}+a \text {. }
$$

All other fields remain the same.

The new system has been analyzed numerically in the case of the log-bell-shaped model. Two typical immune responses are shown in Fig. 4 for $m=3, p=s=1$, $\mu=0.5$. In Fig. 4(a), $\theta_{1}=10^{2}, \theta_{2}=10^{4}$, and localized memory is achieved, whereas in Fig. 4(b), $\theta_{2}$ is increased ten-fold and we now observe percolation. Both parameter settings however fulfill the localization condition [eqn (27)], since they only differ in $\theta_{2}$, and $\theta_{2}$ does not appear in eqn (27). The inequalities such as eqns (21), (23) and (27) are necessary but no sufficient conditions for attainability. This is not surprising since they only deal with the existence and neighborhood stability of the equilibrium-state.

Percolation can develop if $x_{3}$ is activated above threshold by a transiently large value of $x_{2}$. If $x_{3}$ no longer remains virgin, our equilibrium analysis is no longer valid. Instead $x_{3}$ activates $x_{4}$ and an activation cascade can occur [Fig. 4(b)]. In Fig. 5 we present several slices through the six-dimensional $s ; p ; \mu ; \theta_{1} ; \theta_{2} ; k$ parameter space, and identify regions of different qualitative behaviors following perturbation of the virgin-state by a maximal stimulatory dose of antigen given at $t=0\left(a_{0}=\sqrt{\theta_{1} \theta_{2}}\right)$. The alternatives depicted are that the system returns to the (1) 
(a)

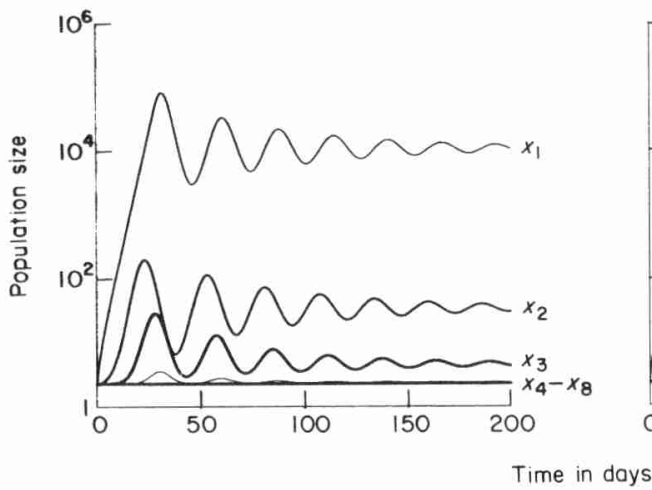

(b)

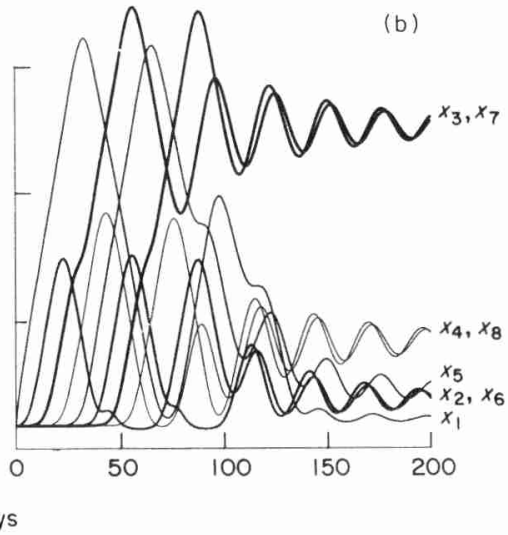

FIG. 4. Immune responses to antigen. A network of eight clones responds to a stimulatory dose of antigen $a_{0}=\sqrt{\theta_{1} \theta_{2}}$ given at $t=0$. Population sizes are shown as a function of time (in days). Parameter setting in (a): $m=3, s=1, \theta_{1}=10^{2}, \theta_{2}=10^{4}, p=1, \mu=0 \cdot 5, k=10^{-5}$; in (b) parameters are identical except that $\theta_{2}=10^{5}$.

virgin-state (white), or (2) attains the localized memory-state for Ab1 (grey), (3) attains other localized activated-states (hatched), or (4) percolates (black).

The figure shows that decreasing the source strengths or increasing the death rate $\mu$ or the stimulation threshold $\theta_{1}$, all of which should facilitate the truth of inequalities (23) and (27), also tends to increase the occurrence of localized memory. Increasing $\mu$ has a major effect that can be seen by comparing the left and the right panels in Fig. 5. We have increased $s$ five-fold in the fourth row of panels; a further increase of $s$ would eliminate the virgin-state as a possible steady-state solution [cf. eqn (6)]. The effect of increasing $\theta_{1}$ depends on $\theta_{2}$ : in each panel there seems to be a linear relation between $\log \left(\theta_{2}\right)$ and the minimal value of $\log \left(\theta_{1}\right)$ that is required for localized memory. Conversely, changing the lattice connectivity $m$, which is also present in conditions (23) and (27), only weakly influences the attainment of localized memory ( $m=2,3,10$ respectively in the three panels at the top).

Parameters that do not appear in the conditions (23) and (27) also influence percolation. Decreasing $\theta_{2}$ corresponds to earlier onset of suppression: this favors localized memory (see Fig. 4 and all panels in Fig. 5). Increasing the rate of antigen elimination ( $k$, i.e. the panels at the bottom of Fig. 5) reduces the attainment of localized memory by increasing the region in which the system returns to the virgin-state. The parameter region where percolation occurs seem to increase if $k$ increases. A number of these observations can be substantiated by algebraic analysis (Weisbuch \& Neumann, submitted to Bull. math. Biol.).

In our analysis we have only concentrated on the sustained stimulation of the Ab1 level. However, in the left bottom panel of Fig. 5 other localized activation states were attained (e.g. at the Ab3 and Ab5 level). These two cases are also shown as time plots in Fig. 6. In a branching tree a set of localized activated-states can be at any antibody level. If $A b_{i}$ is activated, the level beyond, $A b_{i+1}$, is suppressed in a way very similar to the way the Ab2 level is suppressed by the Ab1 clone in the 


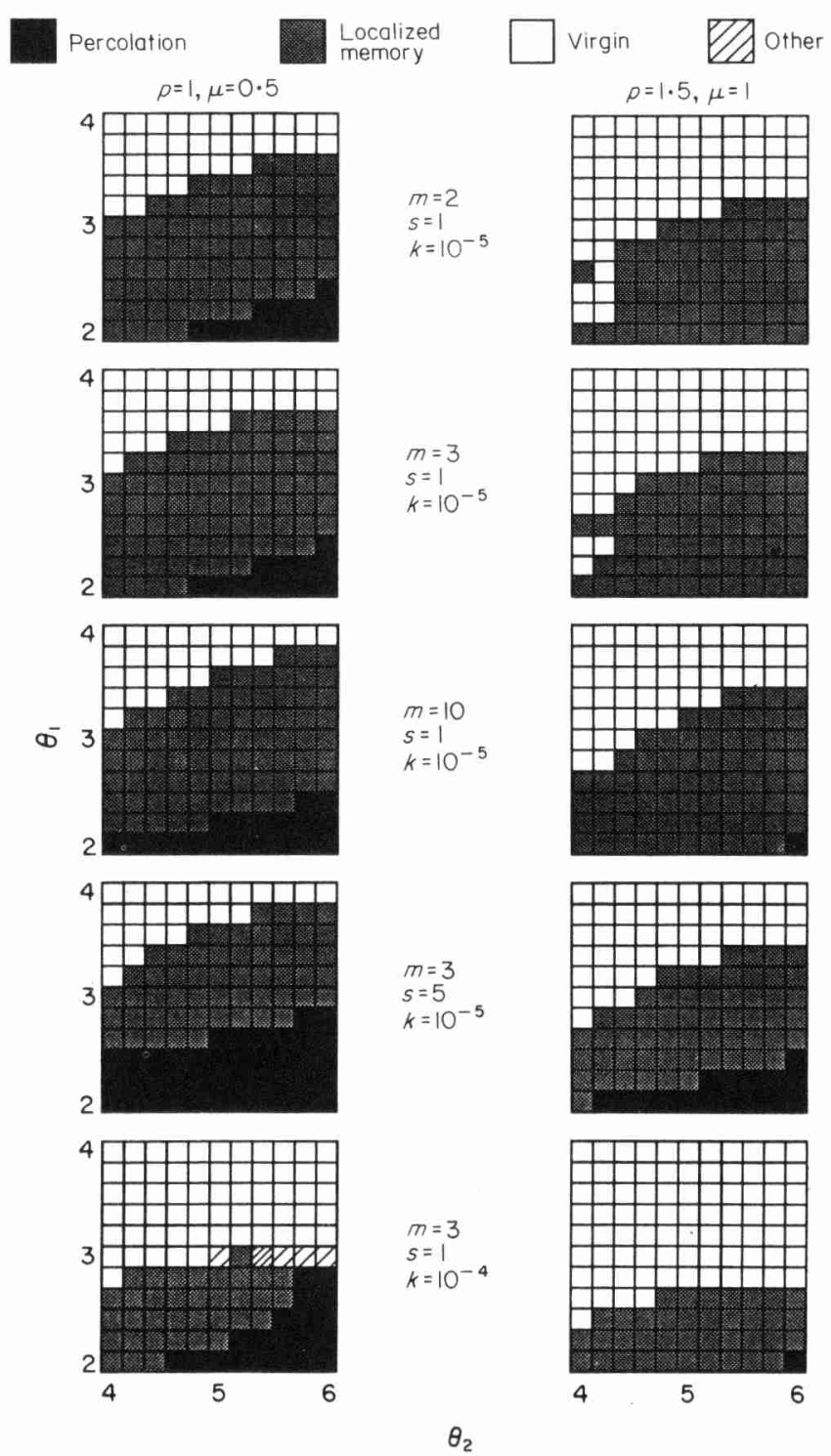

FIG. 5. Conditions for attainment of the virgin-state (white), or of the memory-state (gray), and for percolation (black) after the introduction of antigen. In each panel $\theta_{1}$ and $\theta_{2}$ vary 100 -fold on a logarithmic scale. The shading indicates the behavior that is attained after 500 time steps following a perturbation of the virgin-state with a stimulatory dose of antigen $a_{0}=\sqrt{\theta_{1} \theta_{2}}$. The diagonal shading corresponds to other localized-states, i.e. states in which the memory resides at another level than the Ab1 level (see Fig. 6). The wide diagonal shading corresponds to memory at the Ab3 level, the dense diagonal shading corresponds to memory at the Ab5 level. The left column of panels corresponds to $p=1, \mu=0.5$, the right column corresponds to $p=1 \cdot 5, \mu=1$. From the top to the bottom we vary $m, s$, and $k$ respectively. 

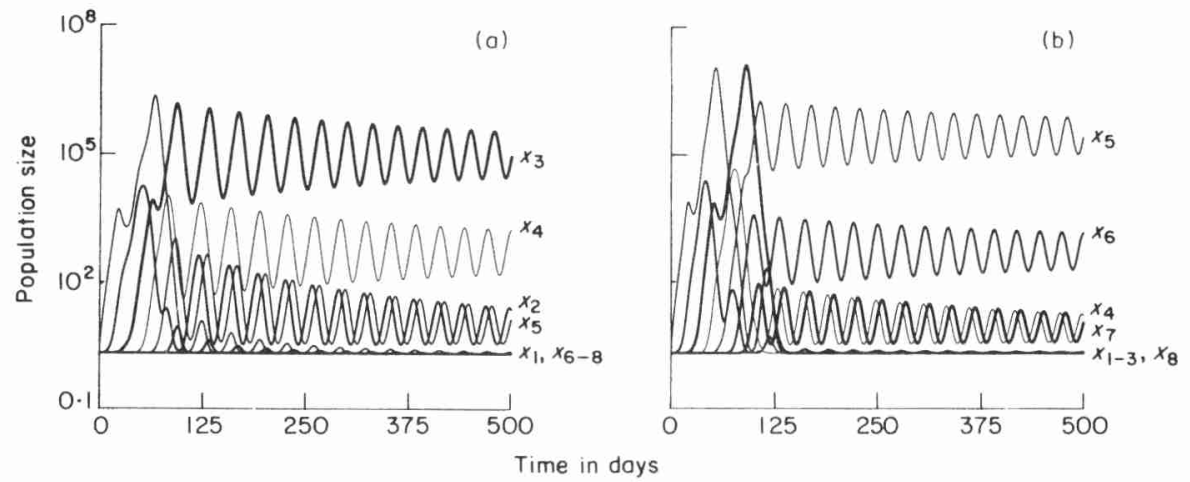

FIG. 6. Attaining other localized activated-states. A network of eight clones responds to a stimulatory dose of antigen $a_{0}=\sqrt{\theta_{1} \theta_{2}}$ given at $t=0$. Population sizes are shown as a function of time (in days). Parameter setting as in Fig. 4 , but $\theta_{1}=10^{3}$ and $\theta_{2}=10^{5}$ in (a) and $\theta_{1}=10^{3}$ and $\theta_{2}=2 \cdot 5 \times 10^{5}$ in (b). In (a) Ab3 attains an immune steady-state, in (b) Ab5 attains an immune steady-state.

localized memory-state. The level below the activated level, $\mathrm{Ab}_{i-1}$, is severely suppressed because it receives $(m-1)$ suppressive inputs. As a consequence that level remains small enough to maintain the virgin-state of the $A b_{i-2}$ level. Activatedstates at various levels can be attained by varying the antigen dose only. Thus, for each setting of the parameters several attractors exist, and the dynamics with its large amplitude transients determine which attractor is attained.

We conclude that both localized memory and percolation occur in this model. We have not yet derived necessary and sufficient conditions on the parameters for attaining localized memory. Suffice to say that Fig. 5 demonstrates that the conditions for attaining the localized memory-state are considerably more severe than those for its existence and local stability [i.e. eqns (23) and (27)].

\section{Discussion}

In this paper we have shown for certain classes of models that if idiotypic memory is maintained by more than one $\mathrm{Ab} 2$, localized memory-states are possible in which only the $\mathrm{Ab} 1$ and the $\mathrm{Ab} 2$ clones are activated. Localization comes about despite the presence of high affinity connections between the activated $\mathrm{Ab} 2 \mathrm{~s}$ and $\mathrm{Ab} 3 \mathrm{~s}$. These results thus provide a counter example to the problem of percolation raised by De Boer \& Hogeweg (1989b). We show that, following perturbation with antigen, both percolation and localized memory are possible dynamical behaviors of this model.

We have described three parameter regimes corresponding to virgin, localized memory, and percolation behaviors. De Boer \& Hogeweg (1989b), studying similar models, missed detecting the second regime, which for their parameters might have been small; see the bottom panels in Fig. 5. Having a simple model that could be analyzed mathematically, in this case the piece-wise linear one, gave us hints to the existence of localized memory-states in the more realistic log bell-shaped model. Thus, even though we do not propose the piece-wise linear model as a realistic 
immunological model, we believe it and other simple models to be valuable in guiding research.

Our main result concerns the existence of localized memories. A consequence of localization is robustness. Introduction of a novel antigen does not interfere with previously established memories as long as the patches of activation of the antigens are sufficiently distinct. In fact, knowing the minimal distance between these islands, one is able to estimate the number of memory islands that could coexist in a network (Weisbuch, 1990). This type of localized memory is different from the distributed memories obtained with neural networks (Hopfield, 1982). In this respect our view differs from that of Varela et al. (1988) and Parisi (1990) who hypothesize that immunological memory is distributed.

Our results are consistent with empirical data on the generation of an anti-idiotypic immune response. These responses are usually observed only if antibody is introduced in a high dose in combination with adjuvant. Indeed, introduction of an Ab1 antibody does not easily lead to an Ab3 response in vivo (Cohen, 1989). Other data however show that in vivo idiotypic cascades do sometimes propagate further than two levels. One example is the studies by Kearney \& Vakil (1986) who can influence adult $\mathrm{Ab} 1$ responses by neonatal manipulation with either Ab2, Ab3, or Ab4. Several immunologists would argue that this reflects a difference between developing and mature immune systems (Coutinho, 1989).

We have shown that for certain regions of the parameter space memory remains localized, and that for other regions percolation occurs due to large transients during the immune response to antigen. The results presented here are only a first step, owing to the considerable simplifications in our models, our limited exploration of the parameter settings, and the uncertainties concerning the antigen removal and dynamics of clonal responses in vivo. The present results at least indicate that idiotypic memory is possible if the immune-state of the $\mathrm{Ab} 1$ is maintained by several $\mathrm{Ab} 2 \mathrm{~s}$.

We thank Geoffrey W. Hoffmann, Lee A. Segel, and Avidan Neumann for helpful suggestions on this paper. This paper was conceived during a visit of RdB to the Ecole Normale Supérieure and a visit of GW to the Santa Fe Institute. This work was supported by the U.S. Department of Energy, the Santa Fe Institute, and by Inserm grant 879002 . The Laboratoire de Physique Statistique de l'ENS is associated with CNRS (URA 1306).

\section{REFERENCES}

COHEN, I. R. (1989). Natural Id-anti-Id networks and the immunological homonculus. In: Theories of Immune Networks (Atlan, H. \& Cohen, I. R., eds) pp. 6-12. Berlin: Springer.

Coutinho, A. (1989). Beyond clonal selection and network. Immunol. Revs. 110, 63-87.

DE BOER, R. J. (1983). GRIND: Great Integrator Differential Equations. Bioinformatics Group, University of Utrecht, The Netherlands.

DE BOER, R. J. (1988). Symmetric idiotypic networks: connectance and switching, stability, and suppression. In: Theoretical Immunology (Perelson, A. S., ed.) Part Two, pp. 265-289, SFI Studies in the Science of Complexity, Vol. III, Redwood City, CA: Addison-Wesley.

DE BOER, R. J. (1989). Extensive percolation in reasonable idiotypic networks. In: Theories of Immune Networks (Atlan, H. \& Cohen, I. R., eds) pp. 26-37. Berlin: Springer.

DE Boer, R. J. \& HogeweG, P. (1989a). Memory but no suppression in low-Dimensional symmetric idiotypic networks. Bull. math. Biol. 51, 223-246. 
De Boer, R. J. \& Hogeweg, P. (1989b). Unreasonable implications of reasonable idiotypic network assumptions. Bull. math. Biol. 51, 381-408.

DE BoER, R. J. \& HogewEG, P. (1989c). Idiotypic network models incorporating T-B cell co-operation: the conditions for percolation. J. theor. Biol. 139, 17-38.

De Boer, R. J., Kevrekidis, I. G. \& Perelson, A. S. (1990). A simple idiotypic network model with complex dynamics. Chem. Eng. Sci. 45, 2375-2382.

Gunther, N. \& HoffmanN, G. W. (1982). Qualitative dynamics of a network model of regulation of the immune system: a rationale for the IgM to IgG switch. J. theor. Biol. 94, 815-855.

Hiernaux, J. (1977). Some remarks on the stability of the idiotypic network. Immunochemistry 14, 733-739.

HOFFMANN, G. W. (1975). A theory of regulation and self-nonself discrimination in an immune network. Eur. J. Immunol. 5, 638-647.

HoffmanN, G. W. (1979). A mathematical model of the stable states of a network theory of selfregulation. In: Systems Theory in Immunology (C. Bruni, Doria, G., Koch, G. \& Strom, R., eds) Vol. 32, pp. 239-257, Lecture Notes in Biomathematics. Berlin: Springer.

HoffMANN, G. W. (1980). On the network theory and H-2 restriction. In: Contemporary Topics Immunobiology (Warner, N. L., ed.) Vol. 11, pp. 185-226. New York: Plenum Press.

Hoffmann, G. W., Kion, T. A., Forsyth, R. B., Soga, K. G., \& Cooper-Willes, A. (1988). The N-dimensional network. In: Theoretical Immunology (Perelson, A. S., ed.) Part Two, pp. 291-319, SFI Studies in the Science of Complexity, Vol. III, Redwood City, CA: Addison-Wesley.

HOPFIELD, (1982). Neural networks and physical systems with emergent collective computational properties. Proc. natn. Acad. Sci. U.S.A. 79, 2554-2558.

JERNE, N. K. (1974). Towards a network theory of the immune system. Ann. Imunol. (Inst. Pasteur) 125C, 373-389.

JERNE, N. K. (1984). Idiotypic networks and other preconceived ideas. Immunol. Revs. 79, 5-24.

KEARNEY, J. F. \& VAKIL, M. (1986). Idiotype-directed interactions during ontogeny play a major role in the establishment of the adult B cell repertoire. Immunol. Rev. 94, 39-50.

PARISI, G. (1990). A simple model for the immune network. Proc. natn. Acad. Sci. U.S.A. 87, 429-433.

Perelson, A. S. (1984). Some mathematical models of receptor clustering by multivalent ligands. In: Cell Surface Dynamics: Concepts and Models (Perelson, A. S., DeLisi, C. \& Wiegel, F. W., eds) pp. 223-275. New York: Marcel Dekker.

PERELSON, A. S. (1988). Towards a realistic model of the immune network. In: Theoretical Immunology (Perelson, A. S., ed.) Part Two, pp. 377-401, SFI Studies in the Science of Complexity, Vol. III, Redwood City, CA: Addison-Wesley.

Perelson, A. S. (1989a). Immune network theory. Immunol. Revs. 110, 5-36.

Perelson, A. S. $(1989 \mathrm{~b})$. Immune networks: a topological view. In: Cell to Cell Signalling: from Experiments to Theoretical Models (Goldbeter, A., ed.) pp. 261-272. London: Academic Press.

Richter, P. H. (1975). A network theory of the immune system. Eur. J. Immunol. 5, 350-354.

Richter, P. H. (1978). The network idea and the immune response. In: Theoretical Immunology (Bell, G. I., Perelson, A. S. \& Pimbley, G. H., eds) pp. 539-569. New York: Marcel Dekker.

Varela, F. J., Coutinho, A., Dupire, B. \& VAZ, N. N. (1988). Cognitive networks: immune, neural, and otherwise. In: Theoretical Immunology (Perelson, A. S., ed.) Part Two, pp. 359-375, SFI Studies in the Science of Complexity, Vol. III. Redwood City, CA: Addison-Wesley.

WeIsBuCH, G. (1989). Dynamical behavior of discrete models of Jerne's network. In: Theories of Immune Networks (Atlan, H. \& Cohen, I. R, eds) pp. 53-62. Berlin: Springer.

WeisbuCH, G. (1990). A shape space approach to the dynamics of the immune system. J. theor. Biol. 143, 507-522. 